\section{Adaptive blind multiuser DS-CDMA downlink equaliser}

\section{Hadef, S. Weiss and M. Rupp}

A robust and simple adaptive blind multiuser equaliser for downlink DS-CDMA systems is presented. The adaptation is based on forcing various user symbols onto a constant modulus, whereby no additional constraints such as the mixed cross-correlation are required. The proposed algorithm has a moderate computational complexity and shows a BER performance close to the MMSE solution.

Introduction: In a DS-CDMA downlink scenario, transmission over a dispersive channel destroys the mutual orthogonality of the codes which are used to multiplex the various users in the system. As a result, the received and code-demultiplexed user signals are subject not only to inter-symbol interference (ISI) owing to channel dispersion but also to multiple access interference (MAI). Consequently the conventional DS-CDMA code-matched filter receiver suffers severe performance degradation, thus motivating the need for better detection strategies.

There have been extensive research efforts to introduce a reliable multiuser detector [1], apt to alleviate the effect of both MAI and ISI While the maximum likelihood (ML) estimation based detector offers the best possible performance [1], its unrealistic complexity renders it unsuitable for downlink applications owing to handset constraints. Alternatively, sub-optimal schemes such as the MMSE multiuser equaliser offer moderate complexity and generally good performance [2]. Recovering several users at the same time in multiuser equalisation exploits more knowledge of the system and has been performed blindly using a constant modulus (CM) criterion [3]. However, in $[3,4]$ additional orthogonality constraints and mutual decorrelation of the recovered user sequence are required.

In this Letter, we derive, based on the definition of a signal model in the following Section, a suitable CM cost function and a stochastic gradient algorithm which is structurally similar to the multiple error filtered-X LMS algorithm in [5]. The addition mutual decorrelation between the various decoded user signals as required in [3] and [4] can be neglected owing to the code-filtering.

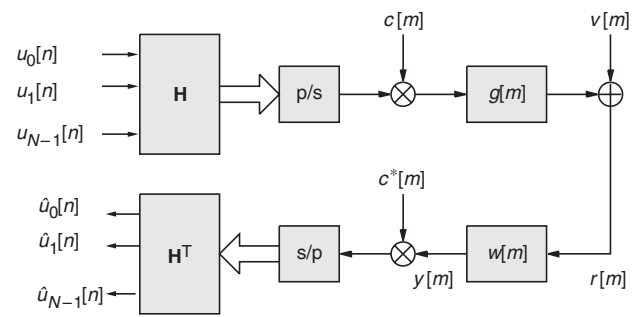

Fig. 1 DS-CDMA downlink signal model

Signal model: We consider the DS-CDMA downlink system in Fig. 1 with multiple symbol-synchronous users, which for simplicity are assumed to have the same rate. The system is fully loaded with $N$ user signals $u_{l}[n], l=0(1) N-1$, which are code multiplexed using Walsh sequences contained in vectors $\mathbf{h}_{l}, l=0(1) N-1$, extracted from an $N \times N$ Hadamard matrix,

$$
\mathbf{H}\left[\mathbf{h}_{0} \mathbf{h}_{1} \ldots \mathbf{h}_{N-1}\right]
$$

The code-multiplexing shown in Fig. 1 is based on a polyphase representation, and could also cover TDMA or FDMA if $\mathbf{H}$ was chosen as an identity or DFT matrix, respectively. The chip rate signal resulting after multiplexing by a parallel-to-serial converter (p/s) is further scrambled by $c[m]$ prior to transmission over a channel with dispersive impulse response $g[m]$ and corruption by additive white Gaussian noise $v[m]$, which is assumed to be independent of the transmitted signal. The dispersive channel $g[m]$ destroys the orthogonality of the Walsh codes, such that direct decoding of the received signal $r[m]$ with descrambling by $c^{*}[m]$ and code-matched filtering by $\mathbf{H}^{\mathrm{T}}$ will lead to MAI and ISI corruption of the decoded user signals $\hat{u}[n]$, $l=0(1) N-1$. To re-establish orthogonality of the codes, a chip rate equaliser $\mathbf{w}$ can be utilised. Therefore, the detected user signals $\hat{u}_{l}[n]$ can be written as

$$
\begin{aligned}
& \hat{u}_{l}[n]=\mathbf{h}_{l}^{\mathrm{T}} \cdot\left[\begin{array}{cc}
c^{*}[n N] & \mathbf{0} \\
c^{*}[n N-1] \\
\ddots \\
\mathbf{0} & c^{*}[n N-N+1]
\end{array}\right] \cdot\left[\begin{array}{c}
y[n N] \\
y[n N-1] \\
\vdots \\
y[n N-n+1]
\end{array}\right] \\
& =\mathbf{w}^{\mathrm{H}} \cdot\left[\begin{array}{cc}
\tilde{\mathbf{h}}_{l}^{\mathrm{T}}[n N] & \mathbf{0} \\
\tilde{\mathbf{h}}_{l}^{\mathrm{T}}[n N] & \ddots \\
\mathbf{0} & \tilde{\mathbf{h}}_{l}^{\mathrm{T}}[n N]
\end{array}\right] \cdot\left[\begin{array}{c}
r[n N] \\
r[n N-1] \\
\vdots \\
r[n N-L-N+2]
\end{array}\right]
\end{aligned}
$$

where the descrambling code $c^{*}[m]$ has been absorbed into a modified and now time-varying code vector $\tilde{\mathbf{h}}_{l}[n N]$, and $\mathbf{w} \in \mathbb{C}^{\mathrm{L}}$ contains the equaliser's $L$ chip-spaced complex conjugate weights. The $l$ th user is thus decoded as

$$
\hat{u}_{l}[n]=\mathbf{w}^{\mathrm{H}} \mathbf{H}_{l}[n N] \mathbf{r}_{n N}
$$

with $\mathbf{H}_{l}[n N] \in \mathbb{Z}^{L \times(N+L-1)}$ being a convolutional matrix comprising the $l$ th user's modified code vector $\tilde{\mathbf{h}}^{\mathrm{T}}[n]$ and $\mathbf{r}_{n N} \in \mathbb{C}^{N+L-1}$. Next, we are concerned with the blind updating of the equaliser vector $\mathbf{w}$.

Blind adaptation: We assume that the user signals $u_{l}[n]$ consist of symbols with a constant modulus $\gamma$, such as BPSK, QPSK, or PAM. Therefore, we would like to blindly adapt the equaliser by forcing all decoded users $\hat{u}_{l}[n]$ onto a constant modulus. This can be formulated, similarly to [3], by a suitable cost function $\xi_{\mathrm{CM}}$,

$$
\xi_{\mathrm{CM}}=\mathcal{E}\left\{\sum_{l=0}^{N-1}\left(\gamma^{2}-\left|\hat{u}_{l}[n]\right|^{2}\right)^{2}\right\}
$$

which measures the deviation of each of the $N$ users' decoded symbols from the desired modulus. Minimising the cost function (4) is ambiguous with a manifold of solutions owing to an indeterminism in phase rotation. However, any member of this manifold is a suitable solution for the equaliser $\mathbf{w}$, and can be used in combination with differential modulation schemes to recover $u_{l}[n]$.

A simple stochastic gradient update rule for $\mathbf{w}$ can be found by calculating the gradient of an instantaneous cost function, i.e. omitting the expectation operator in (4), leading to the stochastic gradient update

$$
\mathbf{w}_{n+1}=\mathbf{w}_{n}-\mu \nabla \hat{\xi}_{\mathrm{CM}}\left(\mathbf{w}_{n}\right)
$$

where $\mu$ is the step size and $n$ the symbol time index. The introduction of gradient noise through inaccurate estimates of the true underlying statistics into the update routine can assist in avoiding the adaptation to remain in flat points of the cost function.

To determine $\nabla \hat{\xi}_{\mathrm{CM}}$, we apply complex vector calculus to (4), yielding

$$
\frac{\partial \hat{\xi}_{\mathrm{CM}}}{\partial \mathbf{w}^{*}}=-2 \sum_{l=0}^{N-1}\left(\gamma^{2}-\left|\hat{u}_{l}[n]\right|^{2}\right) \mathbf{H}_{l}[n N] \mathbf{r}_{n N} \hat{u}_{l}^{*}[n]
$$

This algorithm differs from the standard CM algorithm [6] or its extension in [3] in the inclusion of a code filtered term $\mathbf{H}_{l}[n N] \mathbf{r}_{n N}$ rather than just the equaliser input $\mathbf{r}[n]$. This is structurally similar to a multiple-error filtered-X LMS algorithm [5], where the transfer functions appearing in the paths between the adaptive filter output and the error formations have to be accounted for by modifying the LMS updating scheme. Hence, we refer to the proposed scheme in (6) as the filtered-R multiple error CM algorithm (FIRMER-CMA).

The computational complexity of the proposed algorithm accrues to $2 N^{2} L+2 N L+2 N+L$ multiply accumulates, which is specifically lower than other sub-optimal schemes such as the decorrelator in [7] with $\mathcal{O}\left(N^{3} L^{2}\right)$.

Simulation results: For the simulations below, we apply the FIRMER-CMA for $N=4$ QPSK users to two different sets of channel impulse responses. Both sets include 200 independent fixed channels and have the average magnitude profiles $\left|g_{1}[\mathrm{~m}]\right|$ and $\left|g_{2}[m]\right|$ depicted in Fig. 3. The multipath coefficients of both channel sets are drawn from Rayleigh distributions. Allowing the FIRMER-CMA sufficient time to reach its steady state for each fixed channel realisation, the various BER curves for rotation-corrected systems are compared to their analytic MMSE equaliser perfor- 
mances and to the theoretical performance of QPSK over a nondispersive AWGN channel. The equaliser lengths adopted are $L=20$ and $L=64$ coefficients, for channel sets represented by $\left|g_{1}[\mathrm{~m}]\right|$ and $\left|g_{2}[m]\right|$, respectively. With the middle tap set to unity and an exponentially decreasing step size $\mu=10^{-3}(0: 9999)^{n}$, the algorithm has always been given $10^{3}$ symbol periods to converge prior to correction of the phase rotation and bit error rate (BER) measurement. The BER results are given in Fig. 4 in comparison to the optimal QPSK performance in AWGN and the analytical minimum MSE (MMSE) solution. Note that the FIRMER-CMA closely approaches the MMSE performance.

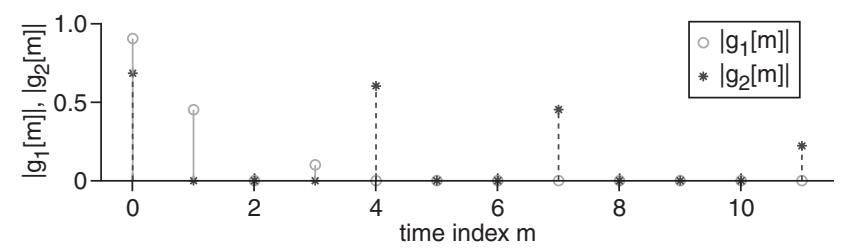

Fig. 2 Average channel impulse response magnitude profiles

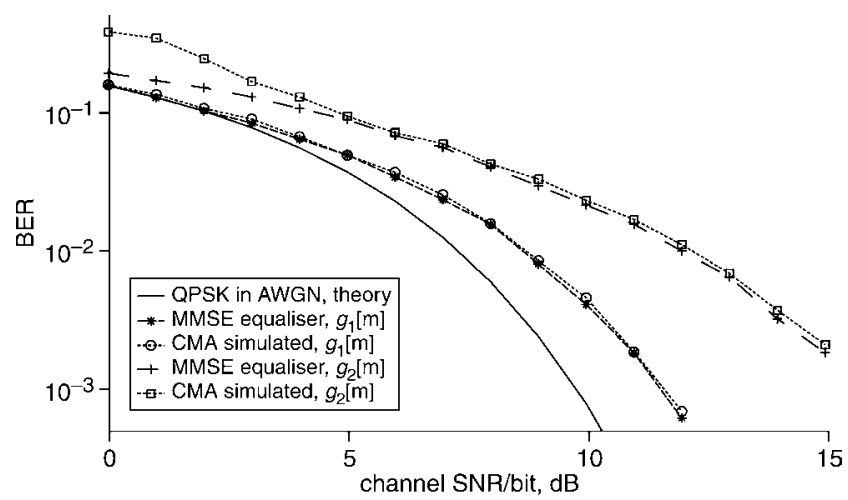

Fig. 3 BER performance of proposed FIRMER-CMA

Conclusion: A blind equalisation approach for a DS-CDMA downlink scenario has been presented, which differs from previous $\mathrm{CM}$ algorithms by a code-prefiltering of its input. Compared to other multiuser CM based solutions in the literature [3, 4], the spreading codes enforce the orthogonality of the decoded user, which renders decorrelation of the various output signals obsolete. The proposed algorithm has a moderate computational complexity. Representative simulations have been presented, highlighting the algorithm's BER performance, which can reach very close to the performance of the MMSE equaliser.

(C) IEE 2005

Electronics Letters online no: 20052902

10 August 2005 doi: 10.1049/el:20052902

M. Hadef and S. Weiss (Department of Electronics \& Computer Science, University of Southampton, Southampton, United Kingdom)

M. Rupp (Institute for Communications \& RF Engineering, Technical University of Vienna, Austria)

E-mail: mh02r@ecs.soton.ac.uk

\section{References}

1 Verdu, S.: 'Multiuser detection' (Cambridge University Press, 1998)

2 Ghauri, I., and Slock, D.T.M.: 'Linear receivers for the DS-CDMA downlink exploiting orthogonality of spreading sequences'. 32nd Asilomar Conf. Signals, Systems and Computers, 1998, Vol. 1, pp. $650-654$

3 Papadias, C., and Paulraj, A.: 'A constant modulus algorithm for multiuser signal separation in presence of delay spread using antenna arrays', IEEE Signal Process. Lett., 1997, 4, (6), pp. 178-181

4 Lambotharan, S., Chambers, J.A., and Constantinides, A.G.: 'Adaptive blind retrieval techniques for multi-user DS-CDMA signals', Electron. Lett., 1999, 35, (9), pp. 693-695

5 Elliott, S.J., Stothers, I.M., and Nelson, P.A.: 'A multiple error LMS algorithm and its application to the active control of sound and vibration', IEEE Trans. Acoust. Speech Signal Process., 1987, 35, (10), pp. 14231434

6 Johnson, C.R., et al.: 'Blind equalization using the constant modulus criterion: a review', Proc. IEEE, 1998, 86, (10), pp. 1927-1950

7 Buehrer, R.M., Correal-Mendoza, N.S., and Woerner, B.D.: 'A simulation comparison of multiuser receivers for cellular CDMA', IEEE Trans. Veh. Technol., 2000, 49, (4), pp. 1065-1085 